\title{
Araneofauna (Arachnida; Araneae) do Parque Estadual do Turvo, Rio Grande do Sul, Brasil
}

\author{
Luciana Regina Podgaiski ${ }^{1,2}$, Ricardo Ott ${ }^{1}$, Everton Nei Lopes Rodrigues ${ }^{1}$, \\ Erica Helena Buckup ${ }^{I} \&$ Maria Aparecida de Leão Marques ${ }^{1}$
}

Biota Neotropica $v 7$ (n2) - http://www.biotaneotropica.org.br/v7n2/pt/abstract?inventory+bn01107022007

\author{
Recebido em 09/11/06 \\ Versão reformulada recebida em 26/02/07 \\ Publicado em 01/05/07
}

\author{
${ }^{1}$ Museu de Ciências Naturais, Fundação Zoobotânica do Rio Grande do Sul, \\ Seção Zoologia de Invertebrados, Aracnologia. \\ Rua Dr. Salvador França, 1427, Jardim Botânico, CEP 90690-000, Porto Alegre, RS, Brasil \\ ${ }^{2}$ Autor para correspondência: Luciana Regina Podgaiski, \\ e-mail:podgaiski@gmail.com,rott@fzb.rs.gov.br,http://www.fzb.rs.gov.br
}

\begin{abstract}
Podgaiski, L.R., Ott, R., Rodrigues, E.N.L., Buckup, E.H. \& Marques, M.A.L. Araneofauna (Arachnida; Araneae) from Parque Estadual do Turvo, Rio Grande do Sul, Brazil. Biota Neotrop. May/Aug 2007 vol. 7 , no. 2 http://www.biotaneotropica.org.br/v7n2/pt/abstract?inventory+bn01107022007. ISSN 1676-0603.

The araneofauna of the Neotropical region can be considered undersurveyed and lacking standardized inventories that can be used as a base for biodiversity studies. Spiders are recognized as important components of forest ecosystems and appear to be good organisms for studies concerning biodiversity standards. A survey was carried out in the Parque Estadual do Turvo, Rio Grande do Sul, Brazil to determine the richness and composition of the spider fauna. The Park is a protection area that includes the only remainder of the Floresta do Alto Uruguai in Brazil. Four expeditions were conducted over the period of two years, in Spring and Autumn (2003-2005). The main sampling technique was the beating tray, sampling the vegetation along the trails inside the park. Additionally, pitfall-traps, Winckler extractors, nocturnal manual collecting and random samplings were used. Shannon-Wiener (H') diversity indices and rarefaction curves were calculated for the beating tray samplings. A total of 8724 spiders belonging to 37 families were collected. Of these, 34\% (2946) were adults, distributed in 31 families, 157 genera and 447 morphospecies. The richest families were Salticidae (23\%) followed by Araneidae (18\%), Theridiidae (16\%) and Thomisidae (9\%). In the beating tray samples 29 families were recorded. The most abundant families (including young individuals) were Salticidae (23\%), Araneidae (18\%), Thomisidae (14\%), Theridiidae (12\%) and Anyphaenidae (12\%). Nineteen species presented a percentage higher than $1 \%$ of the total of collected adult individuals. The most abundant being Thwaitesia affinis O. P.-Cambridge, 1881 (Theridiidae), Tariona sp.1 (Salticidae) and Misumenops argenteus (Mello-Leitão, 1929) (Thomisidae). The Spring periods revealed more adult individuals and species and the Shannon-Wiener (H') diversity was considered higher in the periods of Autumn. The rarefaction curves did not reach asymptote, suggesting the existence of non-sampled species. The spider richness registered in this survey is the greatest observed for Rio Grande do Sul, and the second greatest for Brazil. These information show the importance of this ecosystem for biodiversity conservation in Brazil.

Keywords: spiders, inventory, Neotropical, biodiversity.

\section{Resumo}

Podgaiski, L.R., Ott, R., Rodrigues, E.N.L., Buckup, E.H. \& Marques, M.A.L. Araneofauna (Arachnida; Araneae) do Parque Estadual do Turvo, Rio Grande do Sul, Brasil. Biota Neotrop. May/Aug 2007 vol. 7, no. 2 http://www.biotaneotropica.org.br/v7n2/pt/abstract?inventory+bn01107022007. ISSN 1676-0603.

A região Neotropical é considerada pouco amostrada com relação à sua araneofauna, carecendo de inventários padronizados que possam servir de base para estudos de biodiversidade. As aranhas são consideradas como importantes componentes dos ecossistemas florestais e aparentam ser organismos ideais para estudos de padrões de biodiversidade. Neste estudo foi efetuado um levantamento da riqueza e composição da fauna de aranhas do Parque Estadual do Turvo, Rio Grande do Sul, Brasil, local que protege o único remanescente representativo da Floresta do Alto Uruguai no Brasil. Foram realizadas quatro expedições semestrais, durante dois anos, no outono e na primavera (2003-2005). Como principal técnica de coleta foi empregado o guarda-chuva entomológico, com o qual amostou-se a vegetação arbóreo-arbustiva da borda da floresta nas estradas internas do Parque. Adicionalmente, foram utilizadas armadilhas de solo, extratores de Winckler, coletas manuais noturnas e amostragens aleatórias. Índice de diversidade de Shannon-Wiener (H') e curvas de rarefação foram calculados para as coletas com o guarda-chuva entomológico. Incluindo todos os métodos, foram coletadas 8724 aranhas pertencentes a 37 famílias. Cerca de 34\% (2946) constituíram-se de aranhas adultas, pertencentes a 31 famílias,
\end{abstract}


157 gêneros e 447 morfoespécies. A maior riqueza de espécies foi registrada para Salticidae (23\%), seguida de Araneidae (18\%), Theridiidae (16\%) e Thomisidae (9\%). Nas amostragens com guarda-chuva entomológico foram registradas 29 famílias; as mais abundantes (incluindo jovens) foram: Salticidae (23\%), Araneidae (18\%), Thomisidae (14\%), Theridiidae (12\%) e Anyphaenidae (12\%). Dezenove espécies apresentaram abundância superior a $1 \%$ do total de indivíduos adultos coletados com esta metodologia. As mais abundantes, foram Thwaitesia affinis O. P.-Cambridge, 1881 (Theridiidae), Tariona sp. 1 (Salticidae) e Misumenops argenteus (Mello-Leitão, 1929) (Thomisidae). Nas primaveras registraram-se as maiores abundâncias de adultos e maiores riquezas de espécies; já, a diversidade de Shannon-Wiener (H') foi maior em ambos os períodos de outono, devido ao menor número de singletons registrados. As curvas de rarefação não alcançaram a assíntota, sugerindo a existência de espécies não amostradas. A riqueza de aranhas registrada neste levantamento é a maior já encontrada para o Rio Grande do Sul, e a segunda maior registrada para o Brasil. Estes resultados denotam a importância deste ecossistema na conservação da biodiversidade do país.

Palavras-chave: aranhas, inventário, Neotropical, biodiversidade.

\section{Introdução}

A perda de biodiversidade é um dos grandes problemas ambientais (Wilson 1988, May et al. 1995). Muitos ambientes naturais encontram-se ameaçados, sem nem mesmo ter sua fauna inventariada. Segundo Raizer et al. (2005), a região Neotropical pode ser considerada pouco amostrada com relação á sua araneofauna, carecendo de inventários padronizados que possam servir de base para estudos de biodiversidade.

As aranhas são freqüentemente consideradas como importantes componentes dos ecossistemas florestais (Moulder \& Reichle 1972, Jennings et al. 1990), aparentando ser bons organismos para estudos de padrões de biodiversidade (Platnick 1999), além de serem abundantes e fáceis de serem amostradas (Turnbull 1973). Elas constituem um dos maiores e mais diversificados grupos de animais existentes (Coddington \& Levi 1991) e, no mundo, estão registradas, até o momento, 39490 espécies de aranhas, incluídas em 3642 gêneros e 111 famílias (Platnick 2006). No Rio Grande do Sul, alguns estudos foram efetuados a respeito da composição da fauna de aranhas em ambientes naturais (por exemplo, Indrusiak \& Kotzian 1998, Baldissera et al. 2004, Rodrigues 2005a, b, Ott et al. 2007), porém, diversas regiões do estado ainda permanecem carentes de pesquisas.

O Parque Estadual do Turvo, situado no município de Derrubadas, noroeste do Rio Grande do Sul, Brasil protege o único remanescente representativo e preservado da Floresta do Alto Uruguai no Brasil (Irgang 1979). Esta floresta, que originalmente abrangia grande parte da Bacia do Rio Uruguai, foi intensamente explorada e parcialmente substituída por monoculturas agrícolas, como soja e trigo no Rio Grande do Sul. Na Argentina, ainda encontram-se áreas florestadas com grande representatividade (Parque Provincial de Moconá e Reserva da Biosfera Yabotí). No entanto, estudos de ecologia e diversidade de aranhas são inexistentes neste bioma. O objetivo deste projeto foi efetuar um levantamento da riqueza e composição da fauna de aranhas do Parque Estadual do Turvo.

\section{Material e Métodos}

\section{1. Área de estudo}

O Parque Estadual do Turvo (27 $00^{\prime} \mathrm{S}$ a $27^{\circ} 20^{\prime} \mathrm{S}$ e $53^{\circ} 40^{\prime} \mathrm{W}$ a $54^{\circ} 10^{\prime} \mathrm{W}$ ) (Figura 1 ) possui 17.500 ha e situa-se às margens do Rio Uruguai no município de Derrubadas, Rio Grande do Sul, Brasil. A área enquadra-se como região fitoecológica da Floresta Estacional Decidual do Alto Uruguai (Teixeira et al. 1986). O clima é tipo Cfa, subtropical, conforme classificação de Köppen, no qual as temperaturas médias do mês mais quente (janeiro) são superiores a $22{ }^{\circ} \mathrm{C} \mathrm{e}$ as do mês mais frio (julho) oscilam entre -3 a $18{ }^{\circ} \mathrm{C}$; as precipita- ções são bem distribuídas ao longo do ano, com totais superiores a $1200 \mathrm{~mm}$ (Moreno 1961).

\section{Metodologia}

As amostragens foram semestrais, realizadas durante dois anos, em quatro expedições ao local de estudo (27-31.X.2003 - primavera; 03-07.V.2004 - outono; 19-23.X.2004 - primavera e 26-30.IV.2005 - outono).

O guarda-chuva entomológico foi utilizado nas amostragens quantitativas diurnas (manhã e tarde) para a coleta das aranhas na vegetação arbóreo-arbustiva da borda da floresta nas estradas internas do Parque: estrada para o Porto Garcia (Figura 1a) e estrada para o Salto do Yucumã (Figura 1b). As aranhas foram armazenadas em potes com álcool $80 \%$. Realizaram-se 37 horas de amostragem ( $1 \mathrm{~h}$ x coletor $\mathrm{x}$ área) na primavera de 2003, 43 horas no outono de 2004, 54 horas na primavera de 2004 e 65 horas no outono de 2005, totalizando 199 horas de esforço amostral.

Para a complementação dos dados obtidos com o guarda-chuva entomológico utilizaram-se outras metodologias de coletas (amostragens qualitativas). Foram utilizadas armadilhas de solo consistindo em potes plásticos ( $10 \mathrm{~cm}$ de diâmetro, $15 \mathrm{~cm}$ de profundidade e $500 \mathrm{ml}$ de volume), dispostos dentro de buracos no solo, distantes cerca de dois metros um do outro, em dois transectos dentro da floresta. O líquido conservante (formalina 4\%) cobriu aproximadamente $200 \mathrm{ml}$ do pote. Trinta armadilhas de solo foram dispostas na primavera de 2003, no outono de 2004 e no outono de 2005 e 40, na primavera de 2004. As armadilhas permaneceram quatro dias em campo em todas as datas amostrais. Coletaram-se 20 amostras de serapilheira $(25 \times 25 \mathrm{~cm})$ na primeira data amostral (primavera/ 2003), que foram armazenadas em sacos plásticos e, posteriormente, processadas com extrator de Winckler. Foram realizadas coletas manuais noturnas, sem padronização amostral, com auxílio de lanterna de cabeça, na primavera de 2003, outono e primavera de 2004, capturando-se as aranhas da superfície do solo e da vegetação no interior da floresta. Amostragens aleatórias, que contemplaram oportunidades de coleta casual foram realizadas em todos os períodos de coleta.

As aranhas adultas coletadas foram morfoespeciadas e tombadas na coleção de aranhas do Museu de Ciências Naturais, Fundação Zoobotânica do Rio Grande do Sul, Porto Alegre (curadora E. H. Buckup). Os indivíduos jovens foram identificados somente em famílias.

Para as coletas realizadas com guarda-chuva entomológico, foram realizadas análises estatísticas acerca da composição de aranhas a partir do programa PASt (Paleontological Statistics, versão 1.34) (Hammer \& Harper 2005). Foi estimado índice de diversidade de 

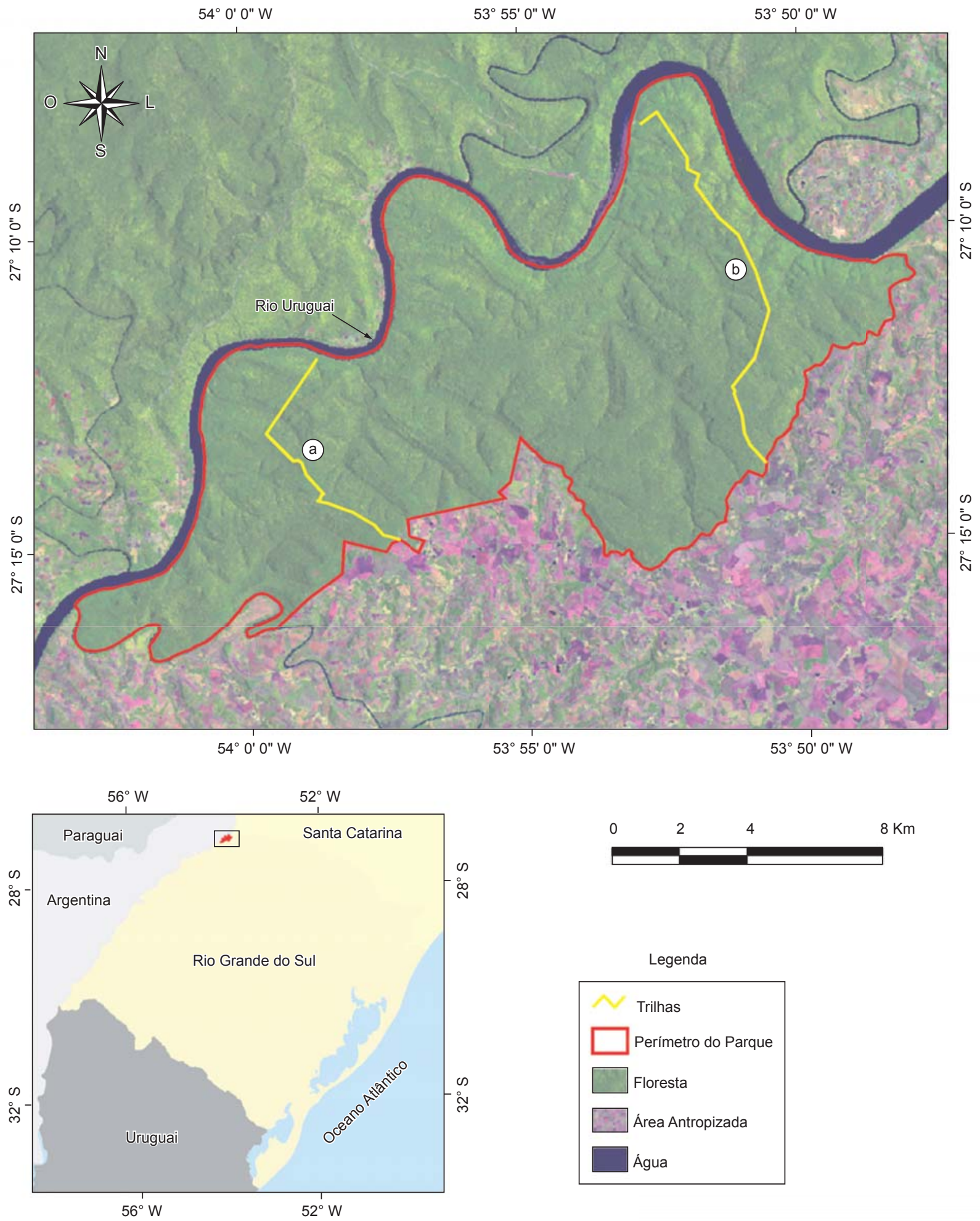

Figura 1. Imagem da área correspondente do Parque Estadual do Turvo, no município de Derrubadas, estado do Rio Grande do Sul, Brasil, mostrando a localização das suas estradas (trilhas) internas - a) Estrada para o Porto Garcia; e b) Estrada para o Salto do Yucumã.

Figure 1. Image of the Parque Estadual do Turvo, in Derrubadas, Rio Grande do Sul, Brazil, showing the trails inside the Park - a) Trail for the Porto Garia; and b) Trail for the Salto do Yucumã. 
Shannon-Wiener (H') para as quatro datas amostrais. Curvas de rarefação foram calculadas para as coletas, verificando a suficiência amostral.

\section{Resultados e Discussão}

Foram coletadas 8724 aranhas, com todas as metodologias, pertencentes a 37 famílias (34 Araneomorphae e três Mygalomorphae). Cerca de 34\% do total (2946) constituíram-se de aranhas adultas, pertencentes a 31 famílias, 157 gêneros e 447 morfoespécies, das quais, 214 espécies são conhecidas nominalmente (48\%) (Tabela 1). As famílias que não apresentaram indivíduos adultos foram Clubionidae, Dipluridae, Hersiliidae, Segestriidae, Theraphosidae e Trechaleidae.

O número de morfoespécies (447) e de famílias de aranhas (37) registrados neste levantamento são bastante expressivos quando comparado com trabalhos realizados em outros biomas do Rio Grande do Sul e do Brasil. Indrusiak \& Kotzian (1998), na região de Santa Maria, Rio Grande do Sul (Floresta Estacional Decidual da Fralda da Serra Geral), registraram 76 morfoespécies em 20 famílias com guardachuva entomológico. Resultado próximo foi encontrado por Rodrigues (2005b), com o mesmo método, em Capão do Leão, Planície Costeira do Rio Grande do Sul (mata de restinga), relacionando 25 famílias e 99 morfoespécies. Na região da Lagoa do Casamento e dos Butiazais de Tapes, Planície costeira do Rio Grande do Sul (mata de restinga), Ott et al. (2007) a partir de coletas com guarda-chuva entomológico, coletas manuais diurnas e noturnas, armadilhas de solo e amostragens de serapilheira, foram capturadas 312 morfoespécies de 33 famílias.

Na região Amazônica, Höfer (1990), em uma floresta de inundação (igapó) perto de Manaus, encontrou 208 morfoespécies representando 37 famílias, utilizando metodologias de amostragem no solo e fotoecletores arbóreos. Para a Reserva Ducke, Amazônia Central, Höfer \& Brescovit (2001), durante aproximadamente 10 anos de coletas, com diversas metodologias e com o acréscimo de informações de outros pesquisadores, contabilizaram 506 morfoespécies e 56 famílias. Na região sudeste do Brasil, Brescovit et al. (2004), também com várias técnicas amostrais (guarda-chuva entomológico, coletas manuais diurnas e noturnas, armadilhas de solo e amostragens de serapilheira), registraram para a Estação Ecológica Juréia-Itatíns, São Paulo, 48 famílias e 274 morfoespécies. Apesar do alto número de espécies registradas para o Parque Estadual do Turvo, percebe-se que o número de famílias de aranhas neste inventário é menor do que os registrados por Höfer \& Brescovit (2001) e Brescovit et al. (2004). Isto pode ser justificado, entre outros fatores, pelo maior esforço amostral realizado por estes autores com métodos que contemplaram a fauna críptica, de solo e serapilheira.

No presente estudo foram contabilizados 165 singletons (37\% das espécies), tendo este percentual se situado abaixo do encontrado em outros trabalhos (Silva \& Coddington 1996 [56\%]; Rodrigues 2005b [41\%]; Raizer et al. 2005 [46\%]).

A maior riqueza de espécies foi encontrada para Salticidae, com 104 morfoespécies (23\%), seguida de Araneidae com 83 (18\%), Theridiidae com 74 (16\%) e Thomisidae com 42 (9\%). Nossos dados corroboram com os resultados encontrados por Höfer \& Brescovit (2001) e Ott et al. (2007), que registraram as mesmas famílias de maior riqueza, nesta mesma ordem de dominância. Flórez (1998), Brescovit et al. (2004) e Raizer et al. (2005) obtiveram Araneidae, Theridiidae e Salticidae respectivamente como famílias de maior riqueza.

\section{Composição da araneofauna amostrada com guarda- chuva entomológico}

As amostragens com guarda-chuva entomológico totalizaram 87\% do total de indivíduos coletados (7598 aranhas; 5286 jovens e 2312 adultos) e $81 \%$ do total de espécies (362 espécies). Com esta metodologia, foram registradas 29 famílias; as mais abundantes (incluindo jovens) foram: Salticidae (23\%), Araneidae (18\%), Thomisidae (14\%), Theridiidae (12\%) e Anyphaenidae (12\%). Nas pesquisas de Indrusiak \& Kotzian (1998) e Rodrigues (2005b), no Rio Grande do Sul, com esta mesma metodologia, foram registradas como mais abundantes as famílias Araneidae, Theridiidae e Anyphaenidae, diferente do padrão encontrado no Parque Estadual do Turvo, possivelmente relacionado às variações da vegetação nos ambientes estudados. Conforme Uetz (1991), a estrutura da vegetação e do habitat influencia diretamente a composição da araneofauna.

Dezenove espécies apresentaram um percentual superior a $1 \%$ da abundância de adultos. As mais abundantes foram Thwaitesia affinis O. P. -Cambridge, 1882 (Theridiidae) e Tariona sp. 1 (Salticidae), ambas ocorrendo em todas as datas amostrais. Misumenops argenteus (Mello-Leitão, 1929) (Thomisidae), terceira espécie mais abundante, ocorreu exclusivamente nas coletas do período de primavera. Outras cinco espécies de abundância superior a $1 \%$ também ocorreram exclusivamente neste período: Lyssomanes leucomelas Mello-Leitão, 1917 (Salticidae); Aysha triunfo Brescovit, 1992 (Anyphaenidae); Patrera procera (Keyserling, 1891) (Anyphaenidae); Wagneriana transitoria (C. L. Koch, 1839) (Araneidae) e Gelanor zonatus (C. L. Koch, 1845) (Mimetidae). Conforme Romero \& Vasconcellos-Neto (2003), é provável que este fato esteja relacionado à época reprodutiva destas espécies, precedendo o verão, período em que pode haver maior quantidade de presas (insetos); no outono, as espécies podem estar representadas, na grande maioria, por imaturos.

Os períodos de primavera demonstraram ser mais abundantes em indivíduos adultos e mais ricos em espécies do que os períodos de outono. Contudo, as amostragens da primavera apresentaram um número muito mais elevado de singletons (espécies representadas por um único indivíduo). Encontraram-se uma média de 60 aranhas por hora de coleta ( 37 jovens e 23 adultos), 214 morfoespécies e 87 singletons na primavera de 2003; 32 aranhas por hora de coleta (16 jovens e 16 adultos), 185 morfoespécies e 82 singletons na primavera de 2004. Com relação às amostragens do outono, foram contabilizadas 37 aranhas por hora de coleta ( 31 jovens e seis adultos), 91 morfoespécies e 52 singletons em 2004 e 31 aranhas por hora de coleta (26 jovens e cinco adultos), 101 morfoespécies e 51 singletons em 2005.

Como o índice de diversidade de Shannon-Wiener (H') combina a riqueza de espécies e a uniformidade (equitabilidade) na distribuição dos indivíduos entre as espécies (Towsend et al. 2006), e atribui um grande peso ás espécies raras, a diversidade da araneofauna foi considerada maior nos períodos de outono (13,6 em 2004 e 11,7 em 2005) do que nos de primavera (11,6 em 2003 e 9,2 em 2004). Isto é justificado, pois os períodos de primavera, apresentaram baixa uniformidade (muitos singletons), apesar de ter tido uma alta riqueza. Os períodos de outono, por sua vez, apresentaram maior uniformidade de espécies (menos singletons), embora menor riqueza.

As curvas de rarefação (Figura 2), para todos os períodos amostrais, na metodologia do guarda-chuva entomológico, não alcançaram a estabilização horizontal, sugerindo a existência de espécies que não foram amostradas no Parque Estadual do Turvo. No entanto, verificase que as curvas dos períodos de primavera apresentaram uma tendência maior à assíntota (Figura 2) com um maior número acumulado de espécies. A riqueza de espécies pode ser comparada entre as datas amostrais considerando-se o mesmo número de indivíduos adultos para todas elas, neste caso, observando-se o valor de abundância relativa mais baixo ( $\mathrm{n}=252$ indivíduos) do outono de 2005 .

Comparando-se as estações, verificou-se que na primavera de 2003 as espécies dominantes foram Thwaitesia affinis (Theridiidae) $(8,4 \%)$ e Misumenops argenteus (Thomisidae) (5,7\%), que juntas somaram $14,1 \%$ dos indivíduos adultos da amostra. No outono 
Tabela 1. Abundância e percentual de aranhas adultas coletadas no Parque Estadual do Turvo, Derrubadas, Rio Grande do Sul, Brasil, em quatro datas amostrais (P.03, primavera 27-31.X.2003; O.04, outono 03-07.V.2004; P.04, primavera 19-23.X.2004; O.05, outono 26-30.IV.2005; A, coleta aleatória; G, guarda-chuva entomológico; N, coleta noturna; S, armadilha de solo; W, extrator de Winckler).

Table 1. Abundance and percentage of adult spiders recorded from Parque Estadual do Turvo, Derrubadas, Rio Grande do Sul, Brazil, in four sampling dates (P.03, Spring 27-31.X.2003; O.04, Autumn 03-07.V.2004; P.04, Spring 19-23.X.2004; O.05, Autumn 26-30.IV.2005; A, random sampling; G, beating tray; N, nocturnal manual collecting; S, pitfall-traps; W, Winckler extractors).

\begin{tabular}{|c|c|c|c|c|c|c|c|}
\hline Família/ Espécie & $\mathbf{P . 0 3}$ & 0.04 & P.04 & 0.05 & Total & $\%$ & Método \\
\hline Mygalomorphae & - & - & - & - & & & \\
\hline Idiopidae & - & - & - & - & 1 & $\mathbf{0 , 0 3}$ & \\
\hline Idiops sp. & - & 1 & - & - & 1 & 0,03 & $S$ \\
\hline Araneomorphae & - & - & - & - & & & \\
\hline Amaurobiidae & - & - & - & - & 11 & 0,37 & \\
\hline Amaurobiidae sp. 1 & 9 & 1 & - & - & 10 & 0,34 & $\mathrm{~S}$ \\
\hline Amaurobiidae sp. 2 & 1 & - & - & - & 1 & 0,03 & $\mathrm{G}$ \\
\hline Anyphaenidae & - & - & - & - & 202 & 6,86 & \\
\hline Aysha triunfo Brescovit, 1992 & 39 & - & 37 & - & 76 & 2,58 & $\mathrm{G}, \mathrm{A}$ \\
\hline Aysha zenzesi (Mello-Leitão,1945) & 4 & - & 14 & - & 18 & 0,61 & $\mathrm{G}, \mathrm{A}$ \\
\hline Aysha sp. 1 & - & - & 1 & - & 1 & 0,03 & $\mathrm{G}$ \\
\hline Aysha sp. 2 & - & 1 & - & - & 1 & 0,03 & G \\
\hline Aysha sp. 3 & - & 1 & - & 1 & 2 & 0,07 & $\mathrm{G}$ \\
\hline Aysha sp. 4 & - & - & 1 & - & 1 & 0,03 & $\mathrm{G}$ \\
\hline Buckupiella imperatriz Brescovit, 1997 & 5 & 1 & 13 & - & 19 & 0,64 & $\mathrm{G}$ \\
\hline Iguarima censoria (Keyserling, 1891) & 7 & - & 3 & - & 10 & 0,34 & $\mathrm{G}, \mathrm{A}, \mathrm{N}$ \\
\hline Jessica glabra (Keyserling, 1891) & 4 & - & 1 & - & 5 & 0,17 & $\mathrm{G}, \mathrm{A}$ \\
\hline Josa sp. & 4 & - & - & - & 4 & 0,14 & $\mathrm{G}$ \\
\hline Katissa sp. & - & - & 1 & - & 1 & 0,03 & $\mathrm{G}$ \\
\hline Patrera procera (Keyserling, 1891) & 22 & - & 19 & - & 41 & 1,39 & $\mathrm{G}, \mathrm{A}, \mathrm{N}$ \\
\hline Patrera sp. & - & 1 & - & - & 1 & 0,03 & $\mathrm{G}$ \\
\hline Teudis sp. & - & - & 1 & - & 1 & 0,03 & G \\
\hline Wulfila albus (Mello-Leitão,1945) & 4 & - & 13 & - & 17 & 0,58 & G \\
\hline Anyphaenidae sp. 1 & - & - & 2 & - & 2 & 0,07 & $\mathrm{~S}$ \\
\hline Anyphaenidae sp. 2 & - & - & 1 & - & 1 & 0,03 & $\mathrm{G}$ \\
\hline Anyphaenidae sp. 3 & 1 & - & - & - & 1 & 0,03 & G \\
\hline Araneidae & - & - & - & - & 424 & 14,4 & \\
\hline Acacesia cornigera Petrunkevitch, 1925 & 4 & - & 2 & 1 & 7 & 0,24 & G \\
\hline Acacesia villalobosi Glueck, 1994 & 2 & - & 2 & - & 4 & 0,14 & $\mathrm{G}, \mathrm{N}$ \\
\hline Acacesia sp. & 1 & - & - & - & 1 & 0,03 & $\mathrm{~N}$ \\
\hline Aculepeira travassosi (Soares \& Camargo, 1948) & 1 & - & - & - & 1 & 0,03 & $\mathrm{G}$ \\
\hline Alpaida albocincta (Mello-Leitão, 1945) & 2 & - & 1 & - & 3 & 0,1 & G \\
\hline Alpaida alto Levi, 1988 & - & 1 & - & - & 1 & 0,03 & $\mathrm{G}$ \\
\hline Alpaida gallardoi Levi, 1988 & - & - & 1 & - & 1 & 0,03 & G \\
\hline Alpaida itapua Levi, 1988 & - & - & 1 & - & 1 & 0,03 & $\mathrm{~N}$ \\
\hline Alpaida lanei Levi, 1988 & - & - & 1 & - & 1 & 0,03 & G \\
\hline Alpaida scriba (Mello-Leitão, 1940) & 1 & - & - & - & 1 & 0,03 & $\mathrm{~N}$ \\
\hline Alpaida septemmammata (O. P. - Cambridge, 1889) & 3 & - & - & - & 3 & 0,1 & $\mathrm{~N}$ \\
\hline Alpaida truncata (Keyserling, 1865) & 1 & - & - & - & 1 & 0,03 & $\mathrm{G}$ \\
\hline Alpaida vanzolinii Levi, 1988 & 1 & - & 1 & - & 2 & 0,07 & $\mathrm{G}$ \\
\hline Alpaida veniliae (Keyserling, 1865) & - & 3 & - & - & 3 & 0,1 & $\mathrm{G}$ \\
\hline Alpaida sp. 1 & - & - & 2 & - & 2 & 0,07 & $\mathrm{~S}$ \\
\hline Alpaida sp. 2 & 1 & - & - & - & 1 & 0,03 & A \\
\hline Araneus guttatus (Keyserling, 1865) & 2 & - & - & - & 2 & 0,07 & $\mathrm{G}$ \\
\hline Araneus iguacu Levi, 1991 & 1 & - & - & - & 1 & 0,03 & $\mathrm{~N}$ \\
\hline Araneus lathyrinus (Holmberg, 1875) & 2 & - & - & - & 2 & 0,07 & $\mathrm{G}, \mathrm{A}$ \\
\hline Araneus omnicolor (Keyserling, 1893) & 1 & - & - & 1 & 2 & 0,07 & $\mathrm{G}, \mathrm{A}$ \\
\hline
\end{tabular}


Tabela 1. Continuação...

\begin{tabular}{|c|c|c|c|c|c|c|c|}
\hline Família/ Espécie & $\mathbf{P . 0 3}$ & 0.04 & P.04 & 0.05 & Total & $\%$ & Método \\
\hline Araneus uniformis (Keyserling, 1879) & 2 & - & 2 & - & 4 & 0,14 & $\mathrm{G}, \mathrm{A}, \mathrm{N}$ \\
\hline Araneus vincibilis (Keyserling, 1893) & 2 & - & 2 & - & 4 & 0,14 & G \\
\hline Argiope argentata (Fabricius, 1775) & 1 & - & - & - & 1 & 0,03 & G \\
\hline Bertrana rufostriata Simon, 1893 & 5 & - & - & - & 5 & 0,17 & G \\
\hline Bertrana striolata Keyserling, 1884 & 1 & - & 3 & 1 & 5 & 0,17 & G \\
\hline Cyclosa bifurcata (Walckenaer, 1842) & - & - & 1 & 1 & 2 & 0,07 & G \\
\hline Cyclosa diversa (O.P.-Cambridge, 1894) & 1 & - & - & - & 1 & 0,03 & G \\
\hline Cyclosa fililineata Hingston, 1932 & 3 & - & - & - & 3 & 0,1 & $\mathrm{G}, \mathrm{N}$ \\
\hline Cyclosa inca Levi, 1999 & & 1 & - & - & 1 & 0,03 & $\mathrm{~N}$ \\
\hline Cyclosa machadinho Levi, 1999 & 2 & - & - & - & 2 & 0,07 & G \\
\hline Cyclosa morretes Levi, 1999 & 2 & - & - & - & 2 & 0,07 & $\mathrm{~N}$ \\
\hline Cyclosa tapetifaciens Hingston, 1932 & 1 & - & - & - & 1 & 0,03 & $\mathrm{~N}$ \\
\hline Eustala sagana (Keyserling, 1893) & 7 & - & 1 & - & 8 & 0,27 & $\mathrm{G}, \mathrm{A}, \mathrm{N}$ \\
\hline Eustala secta Mello- Leitão, 1945 & 2 & - & 6 & - & 8 & 0,27 & G \\
\hline Eustala ulecebrosa (Keyserling, 1892) & 19 & 9 & 19 & 1 & 48 & 1,63 & $\mathrm{G}, \mathrm{A}, \mathrm{N}$ \\
\hline Eustala sp. 1 & - & - & 2 & - & 2 & 0,07 & G \\
\hline Eustala sp. 2 & - & - & 1 & - & 1 & 0,03 & G \\
\hline Eustala sp. 3 & - & - & 1 & - & 1 & 0,03 & G \\
\hline Eustala sp. 4 & 1 & - & - & - & 1 & 0,03 & G \\
\hline Eustala sp. 5 & 1 & - & - & - & 1 & 0,03 & G \\
\hline Eustala sp. 6 & 1 & - & - & - & 1 & 0,03 & G \\
\hline Eustala sp. 7 & 1 & - & 1 & - & 2 & 0,07 & G \\
\hline Eustala sp. 8 & - & - & 3 & - & 3 & 0,1 & $\mathrm{G}, \mathrm{A}$ \\
\hline Hypognatha scutata (Perty, 1833) & 10 & - & 10 & - & 20 & 0,68 & $\mathrm{G}, \mathrm{A}$ \\
\hline Kaira altiventer O. P.-Cambridge, 1889 & - & - & 1 & - & 1 & 0,03 & G \\
\hline Kaira echinus (Simon, 1897) & - & - & 1 & - & 1 & 0,03 & G \\
\hline Kapogea cyrtophoroides (F. O. P.-Cambridge, 1904) & - & - & 1 & - & 1 & 0,03 & G \\
\hline Larinia montecarlo (Levi, 1988) & 4 & 2 & 8 & 3 & 17 & 0,58 & $\mathrm{G}, \mathrm{N}$ \\
\hline Mangora sp. 1 & 7 & 1 & - & 1 & 9 & 0,31 & $\mathrm{G}, \mathrm{N}$ \\
\hline Mangora sp. 2 & 2 & 2 & 1 & 1 & 6 & 0,2 & $\mathrm{G}, \mathrm{N}$ \\
\hline Mangora sp. 3 & 1 & - & 1 & - & 2 & 0,07 & G \\
\hline Mangora sp. 4 & - & - & - & 1 & 1 & 0,03 & G \\
\hline Mangora sp. 5 & - & - & 1 & - & 1 & 0,03 & G \\
\hline Mangora sp. 6 & - & - & - & 1 & 1 & 0,03 & G \\
\hline Manogea porracea (C. L. Koch, 1839) & - & 1 & 3 & 2 & 6 & 0,2 & $\mathrm{G}, \mathrm{N}$ \\
\hline Metazygia gregalis (O. P.-Cambridge, 1889) & - & 2 & - & - & 2 & 0,07 & G \\
\hline Metazygia lagiana Levi, 1995 & - & - & 2 & - & 2 & 0,07 & G \\
\hline Micrathena brevispina (Keyserling, 1864) & 8 & - & 2 & - & 10 & 0,34 & $\mathrm{G}, \mathrm{A}$ \\
\hline Micrathena excavata (C. L. Koch, 1836) & 2 & - & - & - & 2 & 0,07 & G \\
\hline Micrathena flaveola (C. L. Koch, 1839) & 3 & - & 8 & - & 11 & 0,37 & $\mathrm{G}, \mathrm{A}$ \\
\hline Micrathena furva (Keyserling, 1892) & 8 & - & 10 & - & 18 & 0,61 & $\mathrm{G}, \mathrm{A}, \mathrm{N}$ \\
\hline Micrathena jundiai Levi, 1985 & 1 & - & - & 1 & 2 & 0,07 & $\mathrm{G}, \mathrm{A}$ \\
\hline Micrathena nigrichelis Strand, 1908 & - & - & - & 3 & 3 & 0,1 & G \\
\hline Micrathena plana (C. L. Koch, 1836) & 7 & 6 & 4 & - & 17 & 0,58 & $\mathrm{G}, \mathrm{A}, \mathrm{N}$ \\
\hline Micrathena spitzi Mello-Leitão, 1932 & - & 2 & - & - & 2 & 0,07 & $\mathrm{G}, \mathrm{A}$ \\
\hline Ocrepeira galianoae Levi, 1993 & 3 & - & - & - & 3 & 0,1 & G \\
\hline Ocrepeira gnomo (Mello-Leitão, 1943) & 1 & - & 3 & - & 4 & 0,14 & $\mathrm{G}, \mathrm{A}$ \\
\hline Parawixia audax (Blackwall, 1863) & 3 & 1 & 2 & 2 & 8 & 0,27 & $\mathrm{G}, \mathrm{A}$ \\
\hline Parawixia sp. & - & - & 1 & - & 1 & 0,03 & $\mathrm{~N}$ \\
\hline Scoloderus cordatus (Taczanowski, 1879) & - & 10 & 27 & 3 & 40 & 1,36 & $\mathrm{G}, \mathrm{A}$ \\
\hline Scoloderus tuberculifer (O. P.-Cambridge, 1889) & 1 & - & - & - & 1 & 0,03 & G \\
\hline Taczanowskia striata Keyserling, 1879 & 2 & - & - & - & 2 & 0,07 & $\mathrm{G}$ \\
\hline
\end{tabular}


Tabela 1. Continuação...

\begin{tabular}{|c|c|c|c|c|c|c|c|}
\hline Família/ Espécie & $\mathbf{P . 0 3}$ & 0.04 & P.04 & 0.05 & Total & $\%$ & Método \\
\hline Testudinaria lemniscata (Simon, 1893) & - & 1 & - & - & 1 & 0,03 & G \\
\hline Verrucosa meridionalis (Keyserling, 1892) & 8 & - & - & - & 8 & 0,27 & $\mathrm{G}, \mathrm{A}, \mathrm{N}$ \\
\hline Verrucosa sp. 1 & 1 & - & - & - & 1 & 0,03 & G \\
\hline Verrucosa sp. 2 & 6 & 1 & - & - & 7 & 0,24 & $\mathrm{G}, \mathrm{A}, \mathrm{N}$ \\
\hline Wagneriana eupalaestra (Mello-Leitão, 1943) & 6 & - & 6 & - & 12 & 0,41 & $\mathrm{G}, \mathrm{A}, \mathrm{N}$ \\
\hline Wagneriana heteracantha (Mello-Leitão, 1943) & 2 & - & - & - & 2 & 0,07 & $\mathrm{G}, \mathrm{A}$ \\
\hline Wagneriana iguape Levi, 1991 & - & - & 1 & - & 1 & 0,03 & $\mathrm{~N}$ \\
\hline Wagneriana juquia Levi, 1991 & 1 & - & - & - & 1 & 0,03 & $\mathrm{G}$ \\
\hline Wagneriana taim Levi, 1991 & - & 1 & - & - & 1 & 0,03 & G \\
\hline Wagneriana transitoria (C. L. Koch, 1839) & 31 & - & 8 & - & 39 & 1,32 & $\mathrm{G}, \mathrm{A}, \mathrm{N}$ \\
\hline Wagneriana sp. & 4 & - & 6 & - & 10 & 0,34 & $\mathrm{G}, \mathrm{A}, \mathrm{N}$ \\
\hline Caponiidae & - & - & - & - & 11 & 0,37 & \\
\hline Caponina notabilis (Mello-Leitão, 1939) & 1 & - & 5 & - & 6 & 0,2 & $\mathrm{~S}$ \\
\hline Nops meridionalis Keyserling, 1891 & 3 & 2 & - & - & 5 & 0,17 & $\mathrm{~S}$ \\
\hline Corinnidae & - & - & - & - & 67 & 2,27 & \\
\hline Castianeira sp. 1 & - & 8 & - & 3 & 11 & 0,37 & G \\
\hline Castianeira sp. 2 & 1 & - & 2 & - & 3 & 0,1 & $\mathrm{G}, \mathrm{N}, \mathrm{S}$ \\
\hline Castianeira sp. 3 & 1 & 1 & - & 2 & 4 & 0,14 & G \\
\hline Castianeira sp. 4 & - & - & 1 & - & 1 & 0,03 & $\mathrm{~A}$ \\
\hline Corinna bicincta Simon, 1896 & 2 & - & 2 & - & 4 & 0,14 & $\mathrm{G}, \mathrm{A}$ \\
\hline Corinna colombo Bonaldo, 2000 & 1 & 2 & - & 8 & 11 & 0,37 & $\mathrm{G}, \mathrm{A}, \mathrm{N}$ \\
\hline Corinna nitens (Keyserling, 1891) & 1 & - & - & - & 1 & 0,03 & $\mathrm{~N}$ \\
\hline Corinna sp. 1 & - & - & 1 & - & 1 & 0,03 & $\mathrm{~S}$ \\
\hline Corinna sp. 2 & 2 & - & - & - & 2 & 0,07 & $\mathrm{G}, \mathrm{A}$ \\
\hline Creugas lisei Bonaldo, 2000 & - & 1 & - & - & 1 & 0,03 & G \\
\hline Creugas sp. & - & 1 & 2 & - & 3 & 0,1 & $\mathrm{~N}, \mathrm{~S}$ \\
\hline Ianduba varia (Keyserling, 1891) & - & - & 1 & - & 1 & 0,03 & $\mathrm{~S}$ \\
\hline Meriola cetiformis (Strand, 1908) & - & - & - & 1 & 1 & 0,03 & G \\
\hline Paradiestus sp. & - & 3 & - & - & 3 & 0,1 & $\mathrm{G}, \mathrm{N}$ \\
\hline Trachelas robustus Keyserling, 1891 & 4 & 2 & 2 & 1 & 9 & 0,31 & $\mathrm{G}, \mathrm{A}$ \\
\hline Trachelas rugosus Keyserling, 1891 & 4 & - & - & 2 & 6 & 0,2 & $\mathrm{G}, \mathrm{A}, \mathrm{N}$ \\
\hline Trachelas sp. & 2 & 1 & - & - & 3 & 0,1 & $\mathrm{G}$ \\
\hline Trachelopachys keyserlingi (Roewer, 1951) & - & - & 2 & - & 2 & 0,07 & $\mathrm{G}, \mathrm{A}$ \\
\hline Ctenidae & - & - & - & - & 26 & $\mathbf{0 , 8 8}$ & \\
\hline Enoploctenus cyclothorax (Bertkau, 1880) & 1 & - & 1 & - & 2 & 0,07 & $\mathrm{~N}$ \\
\hline Isoctenus sp. 1 & - & 1 & 5 & 2 & 8 & 0,27 & $\mathrm{G}, \mathrm{A}, \mathrm{N}, \mathrm{S}$ \\
\hline Isoctenus $\mathrm{sp} .2$ & - & - & 1 & - & 1 & 0,03 & $\mathrm{~N}$ \\
\hline Oligoctenus ornatus (Keyserling, 1876) & 3 & 3 & 7 & - & 13 & 0,44 & $\mathrm{~A}, \mathrm{~N}$ \\
\hline Phoneutria nigriventer (Keyserling, 1891) & - & - & 2 & - & 2 & 0,07 & $\mathrm{G}, \mathrm{N}$ \\
\hline Deinopidae & - & - & - & - & 2 & 0,07 & \\
\hline Deinopis amica Schiapelli \& Gerschman, 1957 & - & - & - & 2 & 2 & 0,07 & G \\
\hline Dictynidae & - & - & - & - & 19 & 0,64 & \\
\hline Dictynidae sp. 1 & 2 & 3 & 7 & 5 & 17 & 0,58 & G \\
\hline Dictynidae sp. 2 & - & - & 1 & - & 1 & 0,03 & A \\
\hline Dictynidae sp. 3 & - & - & 1 & - & 1 & 0,03 & A \\
\hline Gnaphosidae & - & - & & - & 13 & 0,44 & \\
\hline Zimiromus medius (Keyserling, 1891) & - & - & 1 & - & 1 & 0,03 & G \\
\hline Zimiromus sp. & 1 & - & - & - & 1 & 0,03 & G \\
\hline Gnaphosidae sp. 1 & 1 & 6 & - & - & 7 & 0,24 & $\mathrm{G}, \mathrm{A}$ \\
\hline Gnaphosidae sp. 2 & - & - & 1 & - & 1 & 0,03 & A \\
\hline Gnaphosidae sp. 3 & - & - & 3 & - & 3 & 0,1 & $\mathrm{G}$ \\
\hline
\end{tabular}


Tabela 1. Continuação..

\begin{tabular}{|c|c|c|c|c|c|c|c|}
\hline Família/ Espécie & $\mathbf{P . 0 3}$ & 0.04 & P.04 & 0.05 & Total & $\%$ & Método \\
\hline Linyphiidae & - & - & - & - & 84 & 2,85 & \\
\hline Anodoration claviferum Millidge, 1991 & - & - & - & 2 & 2 & 0,07 & G \\
\hline Dubiaranea sp. 1 & 4 & 1 & 3 & 1 & 9 & 0,31 & $\mathrm{G}, \mathrm{A}$ \\
\hline Dubiaranea sp. 2 & - & - & - & 1 & 1 & 0,03 & G \\
\hline Dubiaranea sp. 3 & 1 & - & - & - & 1 & 0,03 & A \\
\hline Dubiaranea sp. 4 & - & 2 & - & - & 2 & 0,07 & G \\
\hline Erigone sp. & 1 & - & - & - & 1 & 0,03 & $\mathrm{~W}$ \\
\hline Neomaso sp. & 2 & 1 & - & 1 & 4 & 0,14 & $S$ \\
\hline Scolecura cambara Rodrigues, 2005 & - & 1 & 6 & - & 7 & 0,24 & $S$ \\
\hline Sphecozone nigriceps Millidge, 1991 & 13 & 9 & 6 & 4 & 32 & 1,09 & $\mathrm{G}, \mathrm{A}, \mathrm{S}$ \\
\hline Sphecozone novaeteutoniae (Baert, 1987) & 3 & 1 & 7 & 1 & 12 & 0,41 & $\mathrm{G}, \mathrm{S}$ \\
\hline Sphecozone rugosa Millidge, 1991 & - & 1 & - & 1 & 2 & 0,07 & $\mathrm{G}, \mathrm{S}$ \\
\hline Sphecozone sp. & 5 & - & - & - & 5 & 0,17 & $\mathrm{G}, \mathrm{S}$ \\
\hline Linyphiidae sp. 1 & - & - & 1 & - & 1 & 0,03 & $\mathrm{~S}$ \\
\hline Linyphiidae sp. 2 & 3 & - & 1 & - & 4 & 0,14 & $\mathrm{~S}$ \\
\hline Linyphiidae sp. 3 & - & - & 1 & - & 1 & 0,03 & $\mathrm{~S}$ \\
\hline Lycosidae & - & - & - & - & 25 & $\mathbf{0 , 8 5}$ & \\
\hline Aglaoctenus lagotis (Holmberg, 1876) & 1 & - & - & - & 1 & 0,03 & G \\
\hline Hogna gumia (Petrunkevitch, 1911) & 8 & - & - & - & 8 & 0,27 & $\mathrm{~N}$ \\
\hline Lycosidae sp. 1 & 5 & 1 & 1 & 2 & 9 & 0,31 & A, N, S \\
\hline Lycosidae sp. 2 & - & - & 1 & - & 1 & 0,03 & $\mathrm{~N}$ \\
\hline Lycosidae sp. 3 & - & - & - & 2 & 2 & 0,07 & A \\
\hline Lycosidae sp. 4 & - & 1 & - & - & 1 & 0,03 & $\mathrm{~N}$ \\
\hline Lycosidae sp. 5 & 1 & 2 & - & - & 3 & 0,1 & $\mathrm{~A}, \mathrm{~N}$ \\
\hline Mimetidae & & - & - & - & 49 & 1,66 & \\
\hline Ero lata Keyserling, 1891 & 1 & - & 6 & 1 & 8 & 0,27 & $\mathrm{G}, \mathrm{A}, \mathrm{N}$ \\
\hline Gelanor altithorax Keyserling, 1893 & 1 & - & 1 & - & 2 & 0,07 & $\mathrm{~A}, \mathrm{~N}$ \\
\hline Gelanor zonatus (C. L. Koch, 1845) & 23 & - & 14 & - & 37 & 1,26 & $\mathrm{G}, \mathrm{A}, \mathrm{N}$ \\
\hline Mimetus hieroglyphicus Mello-Leitão, 1929 & 1 & - & - & - & 1 & 0,03 & G \\
\hline Mimetidae sp. & - & 1 & & - & 1 & 0,03 & $\mathrm{~N}$ \\
\hline Miturgidae & - & - & - & - & 8 & 0,27 & \\
\hline Cheiracanthium inclusum (Hentz, 1847) & 3 & - & 2 & - & 5 & 0,17 & G \\
\hline Eutichurus ravidus Simon, 1897 & 1 & - & - & - & 1 & 0,03 & $\mathrm{~N}$ \\
\hline Teminius insularis (Lucas, 1857) & - & - & - & 1 & 1 & 0,03 & A \\
\hline Miturgidae sp. & - & - & 1 & - & 1 & 0,03 & $\mathrm{~N}$ \\
\hline Nesticidae & - & - & - & - & 7 & 0,24 & \\
\hline Nesticus brignolii Ott \& Lise, 2002 & 6 & 1 & - & - & 7 & 0,24 & $S$ \\
\hline Ochyroceratidae & - & - & - & - & 3 & 0,1 & \\
\hline Ochyrocera sp. & 2 & - & 1 & - & 3 & 0,1 & $S$ \\
\hline Oonopidae & - & - & - & - & 23 & $\mathbf{0 , 7 8}$ & \\
\hline Neoxyphinus ogloblini Birabén, 1953 & 1 & - & - & - & 1 & 0,03 & $\mathrm{~W}$ \\
\hline Oonops sp. 1 & 1 & 2 & 4 & - & 7 & 0,24 & $\mathrm{G}, \mathrm{S}, \mathrm{W}$ \\
\hline Oonops sp. 2 & - & - & 2 & 2 & 4 & 0,14 & G \\
\hline Oonops sp. 3 & 1 & - & 2 & - & 3 & 0,1 & S \\
\hline Oonops sp. 4 & 3 & - & - & - & 3 & 0,1 & $\mathrm{~S}, \mathrm{~W}$ \\
\hline Oonops sp. 5 & - & 1 & 1 & - & 2 & 0,07 & A, S \\
\hline Orchestina sp. & 1 & 1 & - & - & 2 & 0,07 & G \\
\hline Scaphiella sp. & 1 & - & - & - & 1 & 0,03 & W \\
\hline Oxyopidae & - & - & - & - & 8 & 0,27 & \\
\hline Hamataliva sp. & 1 & - & 1 & 1 & 3 & 0,1 & G \\
\hline Oxyopes salticus Hentz, 1845 & 2 & - & - & - & 2 & 0,07 & G \\
\hline Peucetia sp. & 1 & - & 2 & - & 3 & 0,1 & $\mathrm{G}$ \\
\hline
\end{tabular}


Tabela 1. Continuação...

\begin{tabular}{|c|c|c|c|c|c|c|c|}
\hline Família/ Espécie & P.03 & 0.04 & P.04 & 0.05 & Total & $\%$ & Método \\
\hline Philodromidae & - & - & - & - & 18 & 0,61 & \\
\hline Tibellus sp. & 4 & - & 3 & - & 7 & 0,24 & $\mathrm{G}, \mathrm{A}$ \\
\hline Philodromidae sp. 1 & 1 & - & 5 & - & 6 & 0,2 & G \\
\hline Philodromidae sp. 2 & 4 & - & - & - & 4 & 0,14 & G \\
\hline Philodromidae sp. 3 & - & - & 1 & - & 1 & 0,03 & G \\
\hline Pholcidae & - & - & - & - & 55 & 1,87 & \\
\hline Metagonia argentinensis Mello-Leitão, 1945 & 4 & 1 & 7 & 6 & 18 & 0,61 & $\mathrm{G}, \mathrm{A}$ \\
\hline Mesabolivar luteus (Keyserling, 1891) & 14 & 1 & 14 & 8 & 37 & 1,26 & $\mathrm{G}, \mathrm{N}$ \\
\hline Pisauridae & - & - & - & - & 5 & $\mathbf{0 , 1 7}$ & \\
\hline Staberius spinipes (Taczanowski, 1874) & 1 & - & - & - & 1 & 0,03 & G \\
\hline Pisauridae sp. 1 & 1 & - & 1 & - & 2 & 0,07 & $\mathrm{~N}$ \\
\hline Pisauridae sp. 2 & - & - & 1 & - & 1 & 0,03 & G \\
\hline Pisauridae sp. 3 & 1 & - & - & - & 1 & 0,03 & G \\
\hline Salticidae & - & - & - & - & 747 & 25,4 & \\
\hline Aphirape misionensis Galiano, 1981 & 1 & - & 4 & 17 & 22 & 0,75 & $\mathrm{G}, \mathrm{A}$ \\
\hline Aphirape uncifera (Tullgren, 1905) & - & 14 & - & - & 14 & 0,48 & G \\
\hline Asaphobelis physonychus Simon, 1902 & - & - & 1 & - & 1 & 0,03 & A \\
\hline Asaphobelis sp. 1 & - & 1 & - & - & 1 & 0,03 & G \\
\hline Asaphobelis sp. 2 & - & - & - & 2 & 2 & 0,07 & G \\
\hline Ashtabula sexguttata Simon, 1901 & - & 1 & - & & 1 & 0,03 & G \\
\hline Ashtabula sp. 1 & - & - & - & 1 & 1 & 0,03 & G \\
\hline Ashtabula sp. 2 & - & - & - & 1 & 1 & 0,03 & $\mathrm{G}$ \\
\hline Beata aenea (Mello-Leitão, 1945) & 1 & - & 5 & - & 6 & 0,2 & $\mathrm{G}, \mathrm{A}$ \\
\hline Bellota sp. & - & 1 & - & - & 1 & 0,03 & G \\
\hline Breda apicalis Simon, 1901 & - & - & 2 & - & 2 & 0,07 & G \\
\hline Breda bicruciata (Mello-Leitão, 1943) & - & - & 1 & - & 1 & 0,03 & A \\
\hline Chira distincta Vianna, 1983 & 1 & 4 & - & 11 & 16 & 0,54 & G \\
\hline Chira spinosa (Mello-Leitão, 1939) & - & - & 7 & - & 7 & 0,24 & G \\
\hline Chira sp. & 1 & - & - & - & 1 & 0,03 & G \\
\hline Coryphasia fasciiventris (Simon, 1902) & 3 & - & 3 & - & 6 & 0,2 & $\mathrm{G}, \mathrm{A}$ \\
\hline Coryphasia sp. 1 & 1 & 8 & 12 & 11 & 32 & 1,09 & $\mathrm{G}, \mathrm{A}$ \\
\hline Coryphasia sp. 2 & 5 & 3 & - & - & 8 & 0,27 & $\mathrm{G}, \mathrm{A}$ \\
\hline Cotinusa trifasciata (Mello-Leitão, 1943) & 4 & - & 2 & - & 6 & 0,2 & $\mathrm{G}, \mathrm{A}$ \\
\hline Cotinusa vittata Simon, 1900 & 8 & - & 22 & 4 & 34 & 1,15 & $\mathrm{G}, \mathrm{N}$ \\
\hline Cotinusa sp. 1 & 2 & 1 & 2 & 1 & 6 & 0,2 & G \\
\hline Cotinusa sp. 2 & - & 3 & - & - & 3 & 0,1 & G \\
\hline Cotinusa sp. 3 & - & 1 & - & - & 1 & 0,03 & G \\
\hline Cotinusa sp. 4 & - & - & - & 1 & 1 & 0,03 & G \\
\hline Cotinusa sp. 5 & - & - & 1 & - & 1 & 0,03 & A \\
\hline Encolpius guaraniticus Galiano, 1968 & 2 & - & 1 & - & 3 & 0,1 & $\mathrm{G}, \mathrm{A}, \mathrm{N}$ \\
\hline Euophrys sp. 1 & 2 & - & - & - & 2 & 0,07 & G \\
\hline Euophrys sp. 2 & 2 & - & - & - & 2 & 0,07 & G \\
\hline Gypogyna forceps Simon, 1900 & 1 & - & - & - & 1 & 0,03 & G \\
\hline Ilargus coccineus Simon, 1901 & 1 & - & 1 & 10 & 12 & 0,41 & G \\
\hline Lyssomanes leucomelas Mello-Leitão, 1917 & 14 & - & 47 & - & 61 & 2,07 & $\mathrm{G}, \mathrm{A}, \mathrm{N}$ \\
\hline Lyssomanes miniaceus Peckham \& Wheeler, 1889 & 3 & - & - & - & 3 & 0,1 & G \\
\hline Lyssomanes nigrofimbriatus Mello-Leitão, 1941 & 2 & - & - & - & 2 & 0,07 & $\mathrm{G}, \mathrm{A}$ \\
\hline Lyssomanes pauper Mello-Leitão, 1945 & - & - & 1 & - & 1 & 0,03 & G \\
\hline Lyssomanes tristis Peckham \& Wheeler, 1889 & - & - & 1 & - & 1 & 0,03 & G \\
\hline Mago sp. & - & - & - & 2 & 2 & 0,07 & $\mathrm{G}$ \\
\hline Mopiopia comatula Simon, 1902 & 14 & - & 1 & - & 15 & 0,51 & $\mathrm{G}, \mathrm{A}$ \\
\hline Mopiopia sp. & - & - & 11 & - & 11 & 0,37 & $\mathrm{G}$ \\
\hline
\end{tabular}


Tabela 1. Continuação...

\begin{tabular}{|c|c|c|c|c|c|c|c|}
\hline Família/ Espécie & $\mathbf{P . 0 3}$ & 0.04 & P.04 & 0.05 & Total & $\%$ & Método \\
\hline Neonella sp. & 1 & - & - & - & 1 & 0,03 & W \\
\hline Noegus sp. & 1 & - & - & - & 1 & 0,03 & G \\
\hline Phiale tristis Mello-Leitão, 1945 & 16 & - & 12 & - & 28 & 0,95 & $\mathrm{G}, \mathrm{A}$ \\
\hline Psecas chapoda (Peckham \& Peckham, 1894) & 1 & 1 & 1 & 1 & 4 & 0,14 & $\mathrm{G}, \mathrm{A}$ \\
\hline Sarinda sp. 1 & 4 & - & - & - & 4 & 0,14 & G \\
\hline Sarinda sp. 2 & - & - & 3 & - & 3 & 0,1 & $\mathrm{G}, \mathrm{A}$ \\
\hline Sarinda sp. 3 & 2 & - & - & - & 2 & 0,07 & $\mathrm{G}, \mathrm{N}$ \\
\hline Sassacus helenicus (Mello-Leitão, 1943) & - & - & 2 & - & 2 & 0,07 & G \\
\hline Sassacus sp. 1 & - & - & 1 & - & 1 & 0,03 & G \\
\hline Sassacus sp. 2 & - & - & 1 & - & 1 & 0,03 & G \\
\hline Scopocira histrio Simon, 1900 & 9 & - & 36 & 2 & 47 & 1,6 & $\mathrm{G}, \mathrm{A}$ \\
\hline Semiopyla sp. & 1 & - & - & - & 1 & 0,03 & W \\
\hline Synemosyna aurantiaca (Mello-Leitão, 1917) & 4 & - & 2 & - & 6 & 0,2 & G \\
\hline Synemosyna lauretta Peckham \& Peckham, 1892 & 1 & - & 1 & - & 2 & 0,07 & G \\
\hline Tariona sp. 1 & 28 & 25 & 41 & 39 & 133 & 4,51 & $\mathrm{G}, \mathrm{A}$ \\
\hline Tariona sp. 2 & 2 & - & - & - & 2 & 0,07 & A \\
\hline Tariona sp. 3 & - & 1 & - & - & 1 & 0,03 & G \\
\hline Tariona sp. 4 & - & - & 1 & - & 1 & 0,03 & A \\
\hline Tariona sp. 5 & - & 4 & - & - & 4 & 0,14 & G \\
\hline Tariona sp. 6 & 5 & - & - & - & 5 & 0,17 & G \\
\hline Tariona sp. 7 & 1 & - & - & - & 1 & 0,03 & G \\
\hline Thiodina robusta Mello-Leitão, 1945 & 3 & - & - & - & 3 & 0,1 & G \\
\hline Vinnius uncatus Simon, 1902 & 4 & 3 & 4 & 9 & 20 & 0,68 & $\mathrm{G}, \mathrm{A}$ \\
\hline Wedoquella denticulata Galiano, 1984 & 2 & - & 6 & - & 8 & 0,27 & G \\
\hline Wedoquella macrotheca Galiano, 1984 & 3 & - & 14 & - & 17 & 0,58 & G \\
\hline Wedoquella punctata (Tullgren, 1905) & 7 & - & 4 & - & 11 & 0,37 & G \\
\hline Wedoquella sp. & - & - & 1 & - & 1 & 0,03 & G \\
\hline Yepoella sp. & 1 & 1 & - & 1 & 3 & 0,1 & $\mathrm{G}, \mathrm{S}$ \\
\hline Salticidae sp. 1 & - & - & 4 & - & 4 & 0,14 & G \\
\hline Salticidae sp. 2 & - & - & 1 & - & 1 & 0,03 & G \\
\hline Salticidae sp. 3 & - & - & 1 & - & 1 & 0,03 & G \\
\hline Salticidae sp. 4 & - & - & 1 & - & 1 & 0,03 & G \\
\hline Salticidae sp. 5 & - & 1 & - & - & 1 & 0,03 & $\mathrm{G}$ \\
\hline Salticidae sp. 6 & 6 & - & - & 1 & 7 & 0,24 & $\mathrm{G}, \mathrm{A}$ \\
\hline Salticidae sp. 7 & 1 & - & 4 & 1 & 6 & 0,2 & G \\
\hline Salticidae sp. 8 & 1 & - & - & - & 1 & 0,03 & S \\
\hline Salticidae sp. 9 & 2 & 1 & 1 & - & 4 & 0,14 & $\mathrm{G}, \mathrm{A}$ \\
\hline Salticidae sp. 10 & 4 & 4 & 3 & - & 11 & 0,37 & $\mathrm{G}, \mathrm{A}$ \\
\hline Salticidae sp. 11 & - & - & 1 & - & 1 & 0,03 & G \\
\hline Salticidae sp. 12 & 1 & 8 & 5 & 2 & 16 & 0,54 & G \\
\hline Salticidae sp. 13 & 6 & - & 13 & - & 19 & 0,64 & G \\
\hline Salticidae sp. 14 & - & - & - & 1 & 1 & 0,03 & A \\
\hline Salticidae sp. 15 & - & - & 2 & - & 2 & 0,07 & $\mathrm{G}, \mathrm{A}$ \\
\hline Salticidae sp. 16 & 1 & - & - & - & 1 & 0,03 & G \\
\hline Salticidae sp. 17 & - & - & - & 1 & 1 & 0,03 & G \\
\hline Salticidae sp. 18 & - & 2 & 1 & 3 & 6 & 0,2 & $\mathrm{G}$ \\
\hline Salticidae sp. 19 & - & - & 1 & - & 1 & 0,03 & A \\
\hline Salticidae sp. 20 & 3 & - & 2 & - & 5 & 0,17 & $\mathrm{G}, \mathrm{A}$ \\
\hline Salticidae sp. 21 & 2 & 1 & 2 & - & 5 & 0,17 & $\mathrm{G}, \mathrm{A}$ \\
\hline Salticidae sp. 22 & - & - & 1 & - & 1 & 0,03 & G \\
\hline Salticidae sp. 23 & - & - & 1 & - & 1 & 0,03 & $\mathrm{G}$ \\
\hline Salticidae sp. 24 & 1 & - & - & - & 1 & 0,03 & A \\
\hline
\end{tabular}


Tabela 1. Continuação..

\begin{tabular}{|c|c|c|c|c|c|c|c|}
\hline Família/ Espécie & $\mathbf{P . 0 3}$ & 0.04 & P.04 & 0.05 & Total & $\%$ & Método \\
\hline Salticidae sp. 25 & - & - & - & 1 & 1 & 0,03 & G \\
\hline Salticidae sp. 26 & - & - & 3 & - & 3 & 0,1 & G \\
\hline Salticidae sp. 27 & - & - & - & 1 & 1 & 0,03 & $\mathrm{G}$ \\
\hline Salticidae sp. 28 & 4 & - & 9 & - & 13 & 0,44 & $\mathrm{~S}$ \\
\hline Salticidae sp. 29 & 2 & - & 2 & - & 4 & 0,14 & $\mathrm{G}, \mathrm{A}$ \\
\hline Salticidae sp. 30 & 6 & - & 5 & - & 11 & 0,37 & $\mathrm{~S}$ \\
\hline Salticidae sp. 31 & - & - & 3 & 1 & 4 & 0,14 & G \\
\hline Salticidae sp. 32 & 1 & - & 1 & - & 2 & 0,07 & $\mathrm{~S}$ \\
\hline Salticidae sp. 33 & 1 & - & - & - & 1 & 0,03 & $\mathrm{~S}$ \\
\hline Salticidae sp. 34 & 1 & - & - & - & 1 & 0,03 & G \\
\hline Salticidae sp. 35 & 1 & - & - & - & 1 & 0,03 & $\mathrm{G}$ \\
\hline Salticidae sp. 36 & 1 & - & - & - & 1 & 0,03 & G \\
\hline Salticidae sp. 37 & 1 & - & - & - & 1 & 0,03 & W \\
\hline Salticidae sp. 38 & - & - & 1 & - & 1 & 0,03 & G \\
\hline Scytodidae & - & - & - & - & 23 & 0,78 & \\
\hline Scytodes sp. 1 & 2 & - & - & 7 & 9 & 0,31 & $\mathrm{G}, \mathrm{N}$ \\
\hline Scytodes sp. 2 & 4 & - & 3 & 5 & 12 & 0,41 & $\mathrm{G}, \mathrm{A}$ \\
\hline Scytodes sp. 3 & - & - & 1 & 1 & 2 & 0,07 & $\mathrm{G}, \mathrm{A}$ \\
\hline Selenopidae & - & - & - & - & 8 & 0,27 & \\
\hline Selenops rapax Mello-Leitão, 1929 & - & - & 2 & - & 2 & 0,07 & A \\
\hline Senoculidae & - & - & - & - & 6 & 0,2 & \\
\hline Senocolus sp. 1 & 5 & - & - & - & 5 & 0,17 & $\mathrm{G}, \mathrm{N}$ \\
\hline Senocolus sp. 2 & 1 & - & - & - & 1 & 0,03 & $\mathrm{~A}$ \\
\hline Sparassidae & - & - & - & - & 22 & 0,75 & \\
\hline Olios albus Mello-Leitão, 1918 & 2 & - & - & - & 2 & 0,07 & G \\
\hline Olios caprinus Mello-Leitão, 1918 & 1 & 2 & 5 & 1 & 9 & 0,31 & $\mathrm{G}, \mathrm{A}$ \\
\hline Polybetes rapidus (Keyserling, 1880) & 2 & - & 4 & 2 & 8 & 0,27 & $\mathrm{G}, \mathrm{A}, \mathrm{N}$ \\
\hline Sparassidae sp. & 2 & - & 1 & - & 3 & 0,1 & G \\
\hline Tetragnathidae & - & - & - & - & 102 & 3,46 & \\
\hline Chrysometa boraceia Levi, 1986 & - & - & 1 & - & 1 & 0,03 & $\mathrm{~N}$ \\
\hline Chrysometa ludibunda (Keyserling, 1893) & 15 & 10 & 1 & 10 & 36 & 1,22 & $\mathrm{G}, \mathrm{N}$ \\
\hline Glenognatha lacteovittata (Mello-Leitão, 1944) & 5 & 2 & - & - & 7 & 0,24 & $\mathrm{~A}, \mathrm{~N}$ \\
\hline Leucauge roseosignata Mello-Leitão, 1943 & 7 & 1 & 2 & - & 10 & 0,34 & $\mathrm{G}, \mathrm{A}, \mathrm{N}$ \\
\hline Leucauge volupis (Keyserling, 1893) & 11 & 1 & - & - & 12 & 0,41 & $\mathrm{G}, \mathrm{A}, \mathrm{N}$ \\
\hline Leucauge sp. 1 & 5 & 3 & 2 & 8 & 18 & 0,61 & G \\
\hline Leucauge sp. 2 & 5 & - & - & - & 5 & 0,17 & $\mathrm{G}, \mathrm{A}$ \\
\hline Leucauge sp. 3 & - & - & 3 & - & 3 & 0,1 & $\mathrm{G}, \mathrm{A}$ \\
\hline Mecynometa sp. & 2 & 1 & - & - & 3 & 0,1 & $\mathrm{~A}, \mathrm{~N}$ \\
\hline Tetragnatha nitens (Audouin, 1826) & - & 3 & - & - & 3 & 0,1 & $\mathrm{G}, \mathrm{N}$ \\
\hline Tetragnatha sp. 1 & 1 & - & - & - & 1 & 0,03 & G \\
\hline Tetragnatha sp. 2 & - & 2 & - & - & 2 & 0,07 & $\mathrm{~N}$ \\
\hline Tetragnatha sp. 3 & 1 & - & - & - & 1 & 0,03 & $\mathrm{G}$ \\
\hline Theridiidae & - & - & - & - & 622 & 21,1 & \\
\hline Achaearanea altiventer (Keyserling, 1884) & 6 & 2 & - & - & 8 & 0,27 & G \\
\hline Achaearanea analista Levi, 1963 & 27 & 7 & 36 & 4 & 74 & 2,51 & $\mathrm{G}, \mathrm{A}$ \\
\hline Achaearanea bellula (Keyserling, 1891) & - & 1 & - & - & 1 & 0,03 & $\mathrm{G}$ \\
\hline Achaearanea cinnabarina Levi, 1963 & 3 & - & 1 & - & 4 & 0,14 & G \\
\hline Achaearanea hirta (Taczanowski, 1873) & - & 1 & - & 1 & 2 & 0,07 & $\mathrm{G}$ \\
\hline Achaearanea isana Levi, 1963 & - & - & 3 & 2 & 5 & 0,17 & $\mathrm{G}$ \\
\hline Achaearanea jequirituba Levi, 1963 & 3 & - & - & 1 & 4 & 0,14 & $\mathrm{G}, \mathrm{N}$ \\
\hline Achaearanea passiva (Keyserling, 1891) & 14 & 1 & 1 & 1 & 17 & 0,58 & $\mathrm{G}, \mathrm{N}$ \\
\hline Achaearanea rafaeli Buckup \& Marques, 1991 & 1 & - & - & - & 1 & 0,03 & $\mathrm{G}$ \\
\hline
\end{tabular}


Tabela 1. Continuação..

\begin{tabular}{|c|c|c|c|c|c|c|c|}
\hline Família/ Espécie & $\mathbf{P . 0 3}$ & 0.04 & P.04 & 0.05 & Total & $\%$ & Método \\
\hline Achaearanea tesselata (Keyserling, 1884) & - & - & 2 & - & 2 & 0,07 & G \\
\hline Achaearanea tingo Levi, 1963 & 2 & - & - & - & 2 & 0,07 & G \\
\hline Achaearanea trapezoidalis (Taczanowski, 1873) & 1 & - & - & - & 1 & 0,03 & G \\
\hline Achaearanea triguttata (Keyserling, 1891) & 4 & 4 & 2 & 1 & 11 & 0,37 & $\mathrm{G}, \mathrm{N}$ \\
\hline Achaearanea sp. & 3 & - & 1 & - & 4 & 0,14 & $\mathrm{G}$ \\
\hline Anelosimus studiosus (Hentz, 1850) & 2 & - & - & - & 2 & 0,07 & G \\
\hline Argyrodes elevatus Taczanowski, 1873 & 2 & - & - & - & 2 & 0,07 & G \\
\hline Ariamnes attenuata O. P.-Cambridge, 1881 & 4 & - & 2 & - & 6 & 0,2 & $\mathrm{G}, \mathrm{A}$ \\
\hline Ariamnes sp. & 4 & - & - & - & 4 & 0,14 & $\mathrm{G}, \mathrm{A}$ \\
\hline Chrosiothes niteroi Levi, 1964 & - & 3 & 4 & 1 & 8 & 0,27 & $\mathrm{G}, \mathrm{S}$ \\
\hline Chrosiothes perfidus Marques \& Buckup, 1997 & - & 1 & - & 2 & 3 & 0,1 & G \\
\hline Chrysso nigrosterna Keyserling, 1891 & 17 & 13 & 18 & 2 & 50 & 1,7 & $\mathrm{G}, \mathrm{A}, \mathrm{N}$ \\
\hline Chrysso rubrovittata (Keyserling, 1884) & 6 & - & 1 & 1 & 8 & 0,27 & G \\
\hline Chrysso sp. & - & 3 & - & - & 3 & 0,1 & G \\
\hline Craspedisia cornuta (Keyserling, 1891) & 1 & - & - & - & 1 & 0,03 & G \\
\hline Dipoena alta Keyserling, 1886 & 2 & - & - & - & 2 & 0,07 & $\mathrm{G}, \mathrm{A}$ \\
\hline Dipoena atlantica Chickering, 1943 & - & - & 2 & - & 2 & 0,07 & G \\
\hline Dipoena cordiformis Keyserling, 1886 & 1 & - & - & - & 1 & 0,03 & $\mathrm{~S}$ \\
\hline Dipoena granulata (Keyserling, 1886) & 3 & - & - & - & 3 & 0,1 & $\mathrm{~S}$ \\
\hline Dipoena isthmia Chickering, 1943 & - & - & 1 & 1 & 2 & 0,07 & G \\
\hline Dipoena pumicata (Keyserling, 1886) & 7 & 6 & 3 & 3 & 19 & 0,64 & $\begin{array}{l}\mathrm{G}, \mathrm{N}, \mathrm{S}, \\
\mathrm{W}\end{array}$ \\
\hline Dipoena pusilla (Keyserling, 1886) & 1 & 2 & - & 1 & 4 & 0,14 & G \\
\hline Dipoena santacatarinae Levi, 1963 & 11 & 2 & - & 2 & 15 & 0,51 & G \\
\hline Dipoena taeniatipes Keyserling, 1891 & 3 & 1 & - & - & 4 & 0,14 & $\mathrm{G}, \mathrm{A}$ \\
\hline Dipoena sp. 1 & - & - & 1 & - & 1 & 0,03 & G \\
\hline Dipoena sp. 2 & 1 & - & 3 & - & 4 & 0,14 & G \\
\hline Dipoena sp. 3 & - & - & - & 2 & 2 & 0,07 & G \\
\hline Emertonella taczanowskii (Keyserling, 1886) & 2 & 2 & 1 & 4 & 9 & 0,31 & G \\
\hline Episinus cognatus F. O. P.-Cambridge, 1893 & - & - & 1 & - & 1 & 0,03 & G \\
\hline Euryopis camis Levi, 1963 & 6 & - & 10 & - & 16 & 0,54 & $\mathrm{G}, \mathrm{S}$ \\
\hline Euryopis spinifera (Mello-Leitão, 1944) & - & - & - & 1 & 1 & 0,03 & G \\
\hline Euryopis sp. 1 & - & 1 & - & 1 & 2 & 0,07 & G \\
\hline Euryopis sp. 2 & 1 & - & - & - & 1 & 0,03 & G \\
\hline Faiditus acuminatus (Keyserling, 1891) & 2 & - & - & - & 2 & 0,07 & A \\
\hline Faiditus sp. & 3 & - & 1 & - & 4 & 0,14 & $\mathrm{G}, \mathrm{N}$ \\
\hline Hetschkia gracilis Keyserling, 1886 & 5 & - & 3 & - & 8 & 0,27 & G \\
\hline Neospintharus sp. & - & - & 1 & - & 1 & 0,03 & G \\
\hline Phoroncidia reimoseri Levi, 1964 & 12 & - & 2 & 3 & 17 & 0,58 & $\mathrm{G}, \mathrm{A}, \mathrm{N}$ \\
\hline Rhomphaea altissima Mello-Leitão, 1941 & - & - & 3 & - & 3 & 0,1 & G \\
\hline Rhomphaea brasiliensis Mello-Leitão, 1920 & 2 & - & - & - & 2 & 0,07 & G \\
\hline Rhomphaea sp. 1 & 1 & - & - & - & 1 & 0,03 & G \\
\hline Rhomphaea sp. 2 & - & - & - & 1 & 1 & 0,03 & G \\
\hline Rhomphaea sp. 3 & 1 & - & - & - & 1 & 0,03 & A \\
\hline Styposis selis Levi, 1964 & - & - & 1 & - & 1 & 0,03 & $\mathrm{~S}$ \\
\hline Theridion antron Levi, 1963 & - & - & - & 2 & 2 & 0,07 & G \\
\hline Theridion calcynatum Holmberg, 1876 & 9 & - & 2 & 1 & 12 & 0,41 & $\mathrm{G}, \mathrm{A}$ \\
\hline Theridion filum Levi, 1963 & - & - & 1 & - & 1 & 0,03 & G \\
\hline Theridion opolon Levi, 1963 & - & 1 & - & - & 1 & 0,03 & G \\
\hline Theridion plaumanni Levi, 1963 & 1 & - & 1 & - & 2 & 0,07 & G \\
\hline Theridion positivum Chamberlin, 1924 & - & - & - & 1 & 1 & 0,03 & G \\
\hline Theridion quadripartitum Keyserling, 1891 & 13 & 2 & 10 & 1 & 26 & 0,88 & $\mathrm{G}, \mathrm{A}, \mathrm{W}$ \\
\hline
\end{tabular}


Tabela 1. Continuação..

\begin{tabular}{|c|c|c|c|c|c|c|c|}
\hline Família/ Espécie & $\mathbf{P . 0 3}$ & 0.04 & P.04 & 0.05 & Total & $\%$ & Método \\
\hline Theridion sp. 1 & 2 & - & 1 & - & 3 & 0,1 & G \\
\hline Theridion sp. 2 & 2 & 1 & - & 1 & 4 & 0,14 & G \\
\hline Theridion sp. 3 & 2 & - & - & - & 2 & 0,07 & $\mathrm{G}, \mathrm{N}$ \\
\hline Theridion sp. 4 & 2 & - & - & - & 2 & 0,07 & G \\
\hline Theridion sp. 5 & 1 & 1 & 1 & 2 & 5 & 0,17 & G \\
\hline Theridula puebla Levi, 1954 & 14 & 1 & 7 & - & 22 & 0,75 & G \\
\hline Thwaitesia affinis O. P.-Cambridge, 1882 & 86 & 14 & 49 & 18 & 167 & 5,67 & $\mathrm{G}, \mathrm{A}, \mathrm{N}$ \\
\hline Thymoites anicus Levi, 1964 & 2 & 1 & 2 & - & 5 & 0,17 & $\mathrm{G}$ \\
\hline Thymoites sp. 1 & 3 & 1 & - & - & 4 & 0,14 & G \\
\hline Thymoites sp. 2 & 2 & - & - & - & 2 & 0,07 & G \\
\hline Thymoites sp. 3 & - & - & - & 2 & 2 & 0,07 & $\mathrm{~S}$ \\
\hline Tidarren haemorrhoidale (Bertkau, 1880) & - & - & 1 & - & 1 & 0,03 & $\mathrm{~N}$ \\
\hline Wamba crispulus (Simon, 1895) & 2 & - & 2 & - & 4 & 0,14 & $\mathrm{G}$ \\
\hline Theridiidae sp. & - & 1 & - & - & 1 & 0,03 & $\mathrm{~S}$ \\
\hline Theridiosomatidae & - & - & - & - & 19 & 0,64 & \\
\hline Chthonos sp. & - & - & 1 & - & 1 & 0,03 & G \\
\hline Naatlo splendida (Taczanowski, 1879) & 1 & - & & - & 1 & 0,03 & $\mathrm{~S}$ \\
\hline Naatlo sp. & 3 & - & 7 & - & 10 & 0,34 & G \\
\hline Wendilgarda $\mathrm{sp}$. & 5 & - & - & - & 5 & 0,17 & $\mathrm{G}, \mathrm{A}$ \\
\hline Theridiosomatidae sp. & 1 & 1 & - & - & 2 & 0,07 & $\mathrm{G}, \mathrm{N}$ \\
\hline Thomisidae & - & - & - & - & 295 & 10 & \\
\hline Aphantochilus inermipes Simon, 1929 & 1 & - & - & - & 1 & 0,03 & G \\
\hline Epicadinus sp. & 15 & 4 & 13 & 6 & 38 & 1,29 & $\mathrm{G}, \mathrm{A}$ \\
\hline Epicadus sp. & - & 1 & - & 1 & 2 & 0,07 & G \\
\hline Misumenoides corticatus Mello-Leitão, 1929 & 3 & - & - & - & 3 & 0,1 & G \\
\hline Misumenops argenteus (Mello-Leitão, 1929) & 65 & - & 90 & - & 155 & 5,26 & $\mathrm{G}, \mathrm{A}, \mathrm{N}$ \\
\hline Misumenops pallens (Keyserling, 1880) & 1 & - & 4 & - & 5 & 0,17 & $\mathrm{G}$ \\
\hline Misumenops pallidus (Keyserling, 1880) & - & 1 & - & - & 1 & 0,03 & G \\
\hline Misumenops sp. & 1 & - & - & - & 1 & 0,03 & G \\
\hline Sidymella longispina (Mello-Leitão, 1943) & - & 3 & 1 & 4 & 8 & 0,27 & G \\
\hline Sidymella lucida (Keyserling, 1880) & 1 & - & - & - & 1 & 0,03 & $\mathrm{G}$ \\
\hline Sidymella multispinulosa (Mello-Leitão, 1944) & 1 & - & - & - & 1 & 0,03 & $\mathrm{G}$ \\
\hline Sidymella sp. & - & - & 1 & - & 1 & 0,03 & G \\
\hline Strophius sp. & 2 & - & - & 1 & 3 & 0,1 & $\mathrm{G}$ \\
\hline Synema sp. 1 & 2 & - & - & 3 & 5 & 0,17 & G \\
\hline Synema sp. 2 & - & - & 1 & - & 1 & 0,03 & G \\
\hline Synema sp. 3 & - & - & 1 & - & 1 & 0,03 & G \\
\hline Synema sp. 4 & - & - & 1 & - & 1 & 0,03 & G \\
\hline Synstrophius blanci (Mello-Leitão, 1917) & 1 & - & - & - & 1 & 0,03 & G \\
\hline Tmarus albolineatus Keyserling, 1880 & 4 & - & - & - & 4 & 0,14 & $\mathrm{G}$ \\
\hline Tmarus sp. 1 & - & - & - & 2 & 2 & 0,07 & G \\
\hline Tmarus sp. 2 & - & - & - & 1 & 1 & 0,03 & G \\
\hline Tmarus sp. 3 & - & - & 1 & - & 1 & 0,03 & G \\
\hline Tmarus sp. 4 & 1 & - & - & - & 1 & 0,03 & G \\
\hline Tmarus sp. 5 & 1 & - & - & - & 1 & 0,03 & G \\
\hline Tmarus sp. 6 & 2 & - & 10 & - & 12 & 0,41 & $\mathrm{G}, \mathrm{A}, \mathrm{N}$ \\
\hline Tmarus sp. 7 & 1 & - & - & - & 1 & 0,03 & $\mathrm{G}$ \\
\hline Tmarus sp. 8 & - & - & - & 2 & 2 & 0,07 & $\mathrm{G}$ \\
\hline Tmarus sp. 9 & 1 & - & - & - & 1 & 0,03 & G \\
\hline Tmarus sp. 10 & - & - & 2 & - & 2 & 0,07 & $\mathrm{G}, \mathrm{A}$ \\
\hline Tmarus sp. 11 & 2 & - & - & - & 2 & 0,07 & G \\
\hline Tmarus sp. 12 & - & - & 1 & - & 1 & 0,03 & G \\
\hline
\end{tabular}


Tabela 1. Continuação

\begin{tabular}{|c|c|c|c|c|c|c|c|}
\hline Família/ Espécie & P.03 & 0.04 & P.04 & 0.05 & Total & $\%$ & Método \\
\hline Tobias sp. & 1 & 1 & - & - & 2 & 0,07 & $\mathrm{G}$ \\
\hline Thomisidae sp. 1 & 1 & - & 1 & - & 2 & 0,07 & $\mathrm{G}, \mathrm{A}$ \\
\hline Thomisidae sp. 2 & - & - & 1 & - & 1 & 0,03 & G \\
\hline Thomisidae sp. 3 & 1 & - & - & - & 1 & 0,03 & G \\
\hline Thomisidae sp. 4 & 4 & - & 4 & - & 8 & 0,27 & $\mathrm{G}, \mathrm{A}$ \\
\hline Thomisidae sp. 5 & - & - & - & 1 & 1 & 0,03 & G \\
\hline Thomisidae sp. 6 & - & - & - & 1 & 1 & 0,03 & G \\
\hline Thomisidae sp. 7 & - & - & 1 & - & 1 & 0,03 & G \\
\hline Thomisidae sp. 8 & 1 & - & 6 & - & 7 & 0,24 & G \\
\hline Thomisidae sp. 9 & - & - & 9 & 1 & 10 & 0,34 & $\mathrm{G}, \mathrm{A}$ \\
\hline Thomisidae sp. 10 & 1 & - & - & - & 1 & 0,03 & G \\
\hline Uloboridae & - & - & - & - & 47 & 1,6 & \\
\hline Miagrammopes sp. & 8 & 2 & 7 & 3 & 20 & 0,68 & $\mathrm{G}, \mathrm{A}, \mathrm{N}$ \\
\hline Philoponella fasciata (Mello-Leitão, 1917) & 2 & 1 & 2 & 2 & 7 & 0,24 & G \\
\hline Philoponella sp. & 2 & - & 2 & - & 4 & 0,14 & $\mathrm{G}, \mathrm{N}$ \\
\hline Uloborus trilineatus Keyserling, 1883 & 6 & - & 4 & - & 10 & 0,34 & $\mathrm{G}, \mathrm{A}$ \\
\hline Uloboridae sp. 1 & 1 & - & 1 & - & 2 & 0,07 & G \\
\hline Uloboridae sp. 2 & 2 & - & - & - & 2 & 0,07 & G \\
\hline Uloboridae sp. 3 & 1 & - & 1 & - & 2 & 0,07 & $\mathrm{G}, \mathrm{A}$ \\
\hline Total & 1186 & 313 & 1113 & 334 & 2946 & 100 & \\
\hline
\end{tabular}

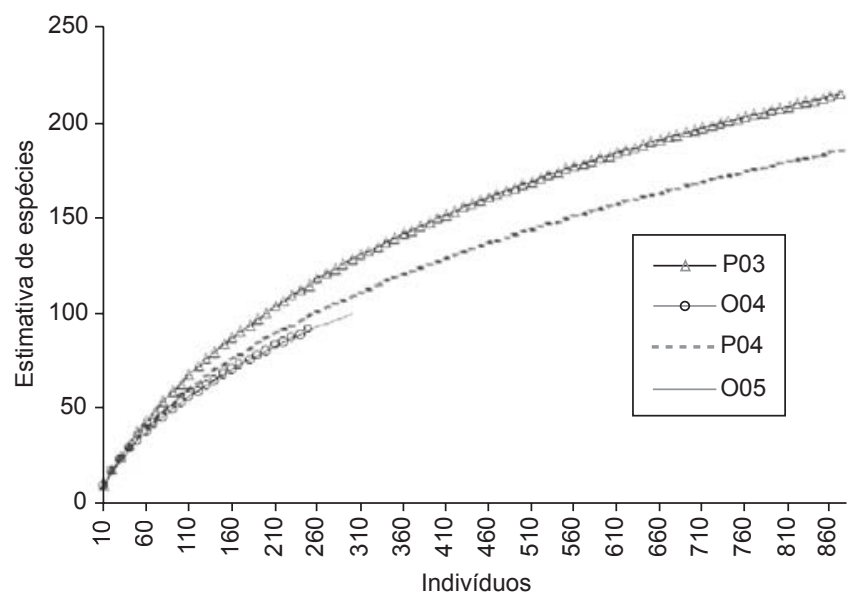

Figura 2. Curvas de rarefação, para a metodologia de guarda-chuva entomológico, em quatro datas amostrais (P.03, primavera 27-31.X.2003; O.04, outono 03-07.V.2004; P.04, primavera 19-23.X.2004; O.05, outono 26-30.IV.2005) no Parque Estadual do Turvo, Derrubadas, Rio Grande do Sul, Brasil.

Figure 2. Rarefaction curves in four sampling dates (P.03, Spring 2731.X.2003; O.04, Autumn 03-07.V.2004; P.04, Spring 19-23.X.2004; O.05, Autumn 26-30.IV.2005), only for beating tray, in Parque Estadual do Turvo, Derrubadas, Rio Grande do Sul, Brazil.

de 2004, duas espécies de Salticidae foram as mais abundantes: Tariona sp. 1 (10\%) e Aphirape uncifera (Tullgren, 1905) (5,7\%). Já na primavera de 2004, sobressaíram-se Misumenops argenteus (Thomisidae) (8,4\%) e Lyssomanes leucomelas (Salticidae) $(5,1 \%)$ e no outono de 2005, Tariona sp. 1 (Salticidae) (12,6\%) e Thwaitesia affinis (Theridiidae) (5,8\%). A ausência de coletas nos períodos do verão e do inverno resultou na perda de informações sobre a composição da comunidade de aranhas nestas estações e do registro de espécies em potencial.

A riqueza de aranhas registrada neste levantamento para a Floresta do Alto Uruguai inserida no Parque Estadual do Turvo é a maior já encontrada para o Rio Grande do Sul, e a segunda maior registrada para o Brasil. Estas informações denotam a importância deste ecossistema para a conservação da biodiversidade do país.

\section{Agradecimentos}

Aos pesquisadores Alexandre Bonaldo (MPEG) pela identificação de exemplares de Corinnidae, Antônio Brescovit (IBSP) pelas identificações de Anyphaenidae e Ctenidae, Éder Álvares (IBSP) pelas identificações de Lycosidae, Cristina Rheims (IBSP) pelas identificações de Sparassidae e pela assessoria no abstract e Arno Lise (PUCRS) pelas identificações de Thomisidae. À Aline Barcellos, Eduardo Borsato, Hilda Alice Gastal, Ingrid Heydrich, Letícia Schmidt, Luciano A. Moura e Tomaz Vital pelo auxílio nas expedições e na coleta de exemplares de aranhas. O presente trabalho foi realizado com o apoio do Conselho Nacional de Desenvolvimento Científico e Tecnológico, CNPq, Brasil.

\section{Referências Bibliográficas}

BALDISSERA, R.; GANADE, G. \& FONTOURA, S. B. 2004. Web spider community response along an edge between pasture and Araucaria forest. Biol. Conserv. 118:403-409.

BRESCOVIT, A. D.; BERTANI, R.; PINTO-DA-ROCHA, R. \& RHEIMS. 2004. Aracnídeos da estação ecológica Juréia-Itatins: Inventário Preliminar e História Natural. In Estação Ecológica Juréia-Itatins (O. A. V. Marques \& W. Dueba, eds.) Holos, Ribeirão Preto, p.198-221.

CODDINGTON, J. A. \& LEVI, H. W. 1991. Systematics and evolution of spiders (Araneae). Annu. Rev. of Ecol. Syst. 22:565-592.

FLÓREZ, D. E. 1998. Estructura de comunidades de arañas (Araneae) en el departamneto del Valle, sur occidente de Colombia. Caldasia 20(2):173-192. 
HAMMER, O. \& HARPER, D. A. T. 2005. PASt: Paleontological Statistics, version 1.34. (Disponível em: http://folk.uio.no/ohammer/past).

HÖFER, H. 1990. The spider community (Araneae) of a central Amazonian blackwater inundation forest (igapó). Acta Zool. Fennica 190:173-179.

HÖFER, H. \& BRESCOVIT, A. D. 2001. Species and guild structure of a Neotropical spider assemblage (Araneae) from Reserva Ducke, Amazonas, Brazil. Andrias 15:99-119.

INDRUSIAK, L. F. \& KOTZIAN, C. B. 1998. Inventário das aranhas arborícolas de três regiões de Santa Maria, RS, Brasil. Revista Ciência e Natura 20:187-214.

IRGANG, B. E. 1979. A mata do Alto Uruguai no Rio Grande do Sul. Ciência e Cultura 32:323-324.

JENNINGS, D. T.; HAEGEN, W. M. V. \& NARAHARA. 1990. A sampling of forest-floor spiders (Araneae) by expellant, Moosehorn National Wildlife Refuge, Maine. J. Arachnol. 18:173-179.

MAY, R. M.; LAWTON, J. H. \& STORK, N. E. 1995. Assessing extinction rates. In Extinction Rates (J. H Lawton \& R. M. May, eds.). Oxford University Press, Oxford, p.1-24.

MOULDER, B. C. \& REICHLE, D. E. 1972. Significance of spider predation in the energy dynamics of forest-floor arthropod communities. Ecol. Monogr. 42:473-498.

MORENO, J. A. 1961. O clima do Rio Grande do Sul. Porto Alegre, Secretaria da Agricultura, 42p.

OTT, R; BUCKUP, E. H. \& MARQUES, M. A. DE L. 2007. 12. Aranhas. In: Biodiversidade da Região da Lagoa do Casamento e dos Butiazais de Tapes, Planície Costeira do Rio Grande do Sul (F. G Becker; R. A. Ramos \& L. de A. Moura, orgs.). Ministério do Meio Ambiente, Brasília, p.172-185.

PLATNICK, N. I. 1999. Dimensions of biodiversity: targeting megadiverse groups. In The living planet in crisis: Biodiversity science and policy (J. Cracraft \& F. T Grifo, eds.). Columbia University Press, New York, p. 33-52.

PLATNICK, N. I. 2006. The world spider catalog, version 7.0. American Museum of Natural History. (Disponível em http://research.amnh.org/ entomology/spiders/catalog/index.html). Acesso em: 28.08.2006.
RAIZER, J.; JAPYASSÚ, H. F., INDICATTI, R. \& BRESCOVIT, A. D. 2005. Comunidade de aranhas (Arachnida: Araneae) do Pantanal Norte (Mato Grosso, Brasil) e sua similaridade com a araneofauna amazônica. Biota Neotropica 5(1a): http://www.biotaneotropica.org.br/v5n1a/pt/ abstract?inventory+BN009051a2005.

RODRIGUES, E. N. L. 2005a. Araneofauna de serapilheira de duas áreas de uma mata de restinga no município de Capão do Leão, Rio Grande do Sul, Brasil. Biotemas 18(1):23-32.

RODRIGUES, E. N. L. 2005b. Fauna araneológica (Arachnida; Araneae) arborícola de duas áreas em uma mata de restinga no sul do Brasil. Acta Biol. Leopold. 27(1):73-92.

ROMERO, G. Q. \& VASCONCELLOS-NETO, J. 2003. Natural history of Misumenops argenteus (Thomisidae): seasonality and diet on Trichogoniopsis adenantha (Asteraceae). J. Arachnol 31:297-304.

SILVA, D. \& CODDINGTON, J. A. 1996. Spiders of Pakitza (Madre de Dios, Peru): Species richness and notes in community structure. In The biodiversity of Pakitza and its environs (D. E. Wilson \& A. Sandoval, eds). Smithsonian Institution, Washington D. C., p.241-299.

TEIXEIRA, M. B.; COURA NETO, A. B.; PASTORE, U. \& RANGEL FILHO, A. L. R. 1986. Vegetação. As regiões Fitoecológicas, sua natureza e seus recursos econômicos. Estudo fitogeográfico. In Levantamento de recursos naturais.Instituto Brasileiro de Geografia e Estatística, Rio de Janeiro, v.33, p.541-632

TOWSEND, C. R.; BEGON, M. \& HARPER, J. L. 2006. Fundamentos de Ecologia. 2aed. Porto Alegre, Artmed Editora. 529p.

TURNBULL, A. L. 1973. Ecology of the true spiders (Araneomorphae). Annu. Ver. Entomol. 18:305-348.

UETZ, G. W. 1991. Habitat structure and spider foraging. In Habitat structure: The physical arrangement of objets in space (S. S. Bell; E. D. McCoy \& H. R. Mushinsky, eds.). Chapman and Hall, London, p.325-348.

WILSON, E. O. 1988. The current state of biological diversity. In Biodiversity (E. O. Wilson, org.). National Academic Press, Washington, D. C., p.3-17.

Título: Araneofauna (Arachnida; Araneae) do Parque Estadual do Turvo, Rio Grande do Sul, Brasil.

Autores: Podgaiski, LR et al.

Biota Neotropica, Vol. 7 (número 2): 2007

http://www.biotaneotropica.org.br/v7n2/pt/abstract?inventory +bn01107022007

Recebido em 0911/06 - Versão reformulada recebida em 26/02/07 - Publicado em 01/05/07

ISSN 1676-0603 